\title{
ESTIMATIVA DA REPETIBILIDADE EM CARACTERES MORFOLÓGICOS E DE PRODUÇÃO DE PALMITO EM PUPUNHEIRA (Bactris gasipaes Kunth) ${ }^{1}$
}

\author{
Nilson César Corrêa Padilha², Maria do Socorro Padilha de Oliveira ${ }^{3}$ e Milton Guilherme da Costa Mota ${ }^{4}$
}

\begin{abstract}
RESUMO - No melhoramento da pupunheira, visando a produção de palmito, é primordial o conhecimento de parâmetros genéticos de caracteres produtivos associados aos morfológicos na seleção de plantas promissoras. Por ser uma palmeira perene com caráter de produção destrutivo, o coeficiente de repetibilidade consiste em uma estratégia desejável. Assim, estimou-se o coeficiente de repetibilidade entre oito caracteres morfológicos e oito de produção de palmito em pupunheiras da coleção de germoplasma da Embrapa Amazônia Oriental. Para tanto, foram avaliadas 65 plantas com variação para número de estipes por planta. O coeficiente de repetibilidade, o número de estipes a serem cortados e o coeficiente de determinação foram obtidos através da análise da variância com um fator de variação, com número variável de observações. Quatro caracteres morfológicos apresentaram coeficientes de repetibilidade variando de 0,5317 a 0,6716 , o que indica regularidade no comportamento das plantas de uma avaliação para outra. No entanto, apenas o comprimento da ráquis foliar mostrou número de medições necessárias para se obter predições com 95\% de confiança. Todos os caracteres produtivos tiveram baixas magnitudes de repetibilidade de $(0,1030$ a 0,2202), o que expressa irregularidades na superioridade das plantas, além de exibirem números de medições elevados. Portanto, o comprimento da ráquis foliar pode ser usado na seleção de pupunheiras para palmito.
\end{abstract}

Palavras-chaves: Planta perene, parâmetros genéticos, seleção e melhoramento genético.

\section{ESTIMATE OF REPEATABILITY IN MORPHOLOGICAL AND HEART OF PALM PRODUCTION CHARACTERS IN PEACH PALM (Bactris gasipaes Kunth)}

\begin{abstract}
Understanding the genetic parameters of the productive characters associated to the morphological is of utmost importance in selecting promising plants for peach palm breeding aiming at heart palm production. Since Bactris gasipaes is a perennial palm tree with a destructive production character, the repeatability coefficient is a desirable strategy to apply. Thus, the repeatability coefficient was estimated among eight morphological characters and eight heart of palm production characters, using peach palm from the Embrapa Eastern Amazon's germ plasm collection. Sixty-five plants were evaluated with different number of stems per plant. Repeatability coefficient, minimum number of stems, and determination coefficients were obtained by means of variance analysis against a variation factor with a variable number of observations. Four morphological characters presented repeatability coefficients ranging from 0.5317 to 0.6716 , showing regularity in plant behavior from one measurement to the other. However, only leaf raquis length showed a number of measurements necessary to allow predictions with 95\% confidence. All the productive characters showed low repeatability scope (0,1030 to 0,2202), indicating irregularities in plant superiority, and also showing high measurement numbers. Therefore, leaf raquis length may be used in the selection of peach palm for heart of palm production.
\end{abstract}

Key words: Perennial tree, genetic parameters, selection and genetic breeding.

1 Recebido para publicação em 2.8.2001.

Aceito para publicação em 9.9.2003.

Parte da dissertação de mestrado apresentada pelo primeiro autor à Faculdade de Ciências Agrárias do Pará.

2 Eng. Florestal, M.S., Faculdade de Ciências Agrárias do Pará, Caixa Postal 917, 66077-530 Belém-PA. ${ }^{3}$ Eng.-Agr., M.S., Embrapa Amazônia Oriental, Caixa Postal 48, 66017-970 Belém-PA, <spadilha@ cpatu.embrapa.br>. ${ }^{4}$ Eng.-Agr. Dr., Faculdade de Ciências Agrárias do Pará, Caixa Postal 917, 66077-530 Belém-PA. 


\section{INTRODUÇÃO}

A pupunheira (Bactris gasipaes Kunth) é uma palmeira perene, nativa da região tropical das Américas, que vem sendo amplamente utilizada (folhas, flores, frutos, caules, palmito e raízes) pelos ameríndios desde a época Pré-colombiana (Camacho, 1972; Clement, 1986). Nos últimos anos essa palmeira vem se destacando na exploração racional de palmito, por apresentar características peculiares como precocidade de produção, rusticidade e capacidade de emitir perfilhamento, garantindo, assim, uma exploração contínua (Clement, 1991).

Na produção de palmito, faz-se necessário o corte do estipe, pois o palmito nada mais é do que o conjunto de folhas em desenvolvimento, envolvido pelas bainhas das folhas mais velhas. Quando se retira a parte superior do estipe, denominado de capitel, ocorre a morte da planta. Desta forma, a utilização do palmito na avaliação dessa espécie leva à destruição da planta, conseqüentemente sua produção precisa ser estimada através de outras características morfológicas para viabilizar e acelerar os programas de melhoramento dessa palmeira. Assim, a busca por características que determinem a produção de palmito tem sido o maior desafio aos melhoristas de palmeiras.

Em pupunheira, estudos de correlações fenotípicas apontam a circunferência do estipe e o número de folhas como caracteres de interesse na seleção de indivíduos desejáveis para palmito, mas para que o processo seja bem-sucedido é necessário que tenha uma alta herdabilidade (Bovi et al., 1992, 1993a).

A determinação da herdabilidade em plantas perenes apresenta dificuldades, principalmente em experimentos com palmeiras, pelo fato de ocuparem grandes áreas e de a maioria deles estar instalada sem delineamentos experimentais. Em vista disso, uma das alternativas dos melhoristas tem sido a obtenção do coeficiente de repetibilidade. Este método define o limite superior do valor do coeficiente de herdabilidade $\left(\mathrm{h}^{2}\right)$ e o grau de determinação genética do caráter em estudo, permitindo também determinar quantas observações fenotípicas devem ser efetuadas em cada indivíduo para que a seleção seja praticada com eficiência e com menor trabalho (Falconer, 1987).

Para Vencovsky (1977), o coeficiente de repetibilidade é muito utilizado no estudo de caracteres de plantas perenes, por se expressar várias vezes ao longo de sua

R. Árvore, Viçosa-MG, v.27, n.4, p.435-442, 2003 vida, e baseia-se na tomada de várias observações no mesmo indivíduo. Portanto, ao escolher um determinado genótipo, espera-se que sua superioridade inicial continue constante durante todo o período de vida. Cruz \& Regazzi (1997) ressaltam a necessidade do bom desempenho em algumas estruturas, ou partes que compõem o indivíduo, de modo a fornecer o potencial do genótipo a ser utilizado como um todo. Para os autores, elevados valores de coeficiente de repetibilidade indicam que é possível predizer o valor real do indivíduo com um número relativamente pequeno de medições.

Estimativas de coeficiente de repetibilidade têm sido realizadas para várias culturas perenes, como no coqueiro (Shrikhande, 1957; Siqueira, 1982); no cupuaçuzeiro (Fonseca et al., 1990; Costa et al., 1997), na seringueira (Gonçalves et al., 1982; Vasconcellos et al., 1985; Gonçalves et al., 1990), em pinheiros (Cornacchia et al., 1995) e em forrageiras (Ferreira et al., 1999). Porém, não há relatos de estudos dessa natureza para palmeiras produtoras de palmito.

Os objetivos deste trabalho foram estimar a repetibilidade para os caracteres morfológicos e de produção de palmito e determinar o número necessário de estipes a serem cortados para uma seleção mais eficiente em pupunheira.

\section{MATERIAL E MÉTODOS}

Este trabalho foi desenvolvido na coleção de germoplasma de pupunheira da Embrapa Amazônia Oriental, em Belém-PA. Nesta coleção há 95 indivíduos (progênies de polinização livre), de 14 anos de idade, em plena fase reprodutiva e com variações para perfilhamento. Assim, para não ocasionar danos à coleção foram estabelecidas algumas condições para a avaliação de palmito, tendo participado do corte apenas os indivíduos com número de estipes por planta (NEP) maior ou igual a 5 e que possuíam circunferência do estipe à altura do peito (CAP) maior ou igual a $22 \mathrm{~cm}$.

Das plantas existentes, 65 apresentaram condições para extração de palmito e permitiram o corte de 171 estipes, nos quais foram feitas as mensurações de caracteres, oito morfológicos e oito de produção do palmito, em dezembro de 1999.

Os caracteres morfológicos foram avaliados no campo, sendo eles: circunferência do estipe, em centímetros (CAP); comprimento de cinco entrenós, em 
centímetros (CEN); altura do estipe mensurada do ponto de inserção das raízes até o início do capitel das folhas, em metros (AE); número de folhas vivas, em unidades (NF); comprimento da bainha foliar das folhas mais externas, em centímetros $(\mathrm{CBF})$; comprimento da ráquis foliar da folha mais externa, em metros (CRF); comprimento do folíolo central da folha mais externa, em centímetros (CFC); e números de pares de folíolos da folha mais externa, em unidades (NPFO).

Os caracteres produtivos foram mensurados no laboratório de fitomelhoramento dessa instituição e envolveram: peso da cabeça do palmito, em gramas (PCP); diâmetro do ápice do palmito, em milímetros (DAP); diâmetro do meio do palmito, em milímetros (DMP); diâmetro da base do palmito, em milímetros (DBP); peso do palmito bruto ou total, em gramas (PPT); peso do palmito creme ou tipo exportação, em gramas (PPC); comprimento do palmito bruto, em centímetros (CPB); e número de toletes de palmito, em unidades (NTP). Para o caráter peso da cabeça do palmito (PCP), desembainhou-se previamente o palmito, deixando de quatro a cinco bainhas.

As estimativas dos componentes de variância e de repetibilidade foram obtidas pelo método da análise de variância (ANOVA) com um fator de variação e número variável de observações, conforme adaptação de Cruz \& Regazzi (1997). Para atender às pressuposições da análise de variância, os caracteres expressos em unidades (NF, NPFO e NTP) com efeitos não-aditivos foram transformados em $\mathrm{x}^{1 / 2}$. $\mathrm{O}$ ajuste para o número de repetições (observações) foi feito através do "K", expresso a seguir:

$$
K=N-\left(\sum n_{\mathrm{i}}^{2} / N\right) / p-1
$$

em que $K=$ fator para diferentes números de medições; $N=$ número de indivíduos cortados; $n=$ número de estipe cortado em cada planta; e $p=$ número de plantas.

\section{RESULTADOS E DISCUSSÃO}

Foram observadas diferenças significativas por meio do teste $\mathrm{F}$ para todos os caracteres morfológicos $(\mathrm{P} \leq 0,01)$, o que fornece indícios da existência de variabilidade ou de heterogeneidade entre as plantas, com possibilidades de auxiliar na identificação de indivíduos desejáveis para produção de palmito (Quadro 1). Pelo fato de as diferenças encontradas serem em decorrência de efeitos fenotípicos, há a necessidade de estimar a expressão do valor genotípico dessas variáveis, de modo que possam ser empregadas com sucesso no melhoramento da pupunheira para palmito.

Em média, as pupunheiras cortadas apresentaram características morfológicas desejáveis para a produção de palmito, ou seja, estipes grossos, com 37,09 cm de circunferência, que está bem acima da desejável para exploração de palmito (CAP $\geq 22 \mathrm{~cm})$, havendo, então, a possibilidade de produzir palmito de primeira qualidade. As folhas também mostraram bainha e ráquis compridas, atingindo $85,47 \mathrm{~cm}$ e $2,49 \mathrm{~m}$, respectivamente, o que é importante nesse tipo de exploração.

Quadro 1 - Análise da variância para oito caracteres morfológicos de 65 plantas de pupunheira da coleção de germoplasma da Embrapa Amazônia Oriental, Belém-PA, 2001

Table 1 - Variance analysis for eight morphological characters of 65 peach palm plants of Embrapa Eastern Amazon germ plasm collection, Belém, PA, 2001

\begin{tabular}{|c|c|c|c|c|}
\hline \multirow{2}{*}{ Caráter } & \multicolumn{2}{|c|}{ Quadrados Médios } & \multirow{2}{*}{ Média } & \multirow{2}{*}{$\begin{array}{l}\mathrm{CV} \\
(\%)\end{array}$} \\
\hline & Planta & Erro & & \\
\hline Circunferência do estipe $(\mathrm{cm})$ & $49,9982 * *$ & 21,3077 & 37,09 & 12,44 \\
\hline Comprimento dos entrenós $(\mathrm{cm})$ & $1.202,4279 * *$ & 288,8469 & 65,59 & 25,91 \\
\hline Altura do estipe (m) & $7,6641 * *$ & 2,4725 & 5,50 & 28,56 \\
\hline Número de folhas ${ }^{\prime}$ (unid.) & $0,3637 * *$ & 0,1701 & 3,11 & 13,24 \\
\hline Comprimento da bainha foliar $(\mathrm{cm})$ & $567,5560 * *$ & 201,5187 & 85,47 & 16,61 \\
\hline Comprimento da ráquis foliar $(\mathrm{m})$ & $0,2944 * *$ & 0,0466 & 2,49 & 8,67 \\
\hline Comprimento do folíolo central $(\mathrm{cm})$ & $157,4457 * *$ & 39,8441 & 62,33 & 10,13 \\
\hline Número de pares de folíolos ${ }^{\underline{1}}$ (unid.) & $1,0728 * *$ & 0,2651 & 10,12 & 5,08 \\
\hline
\end{tabular}

** significativo a $1 \%$ de probabilidade pelo teste $\mathrm{F} \mathrm{e}^{1 /}$ valores transformados em $\mathrm{x}^{1 / 2}$. 
Os coeficientes de variação obtidos para essas características estiveram dentro do limite de experimentos avaliados em campo, evidenciando boa precisão experimental. As características que exibiram os maiores valores foram o comprimento dos entrenós (CEN) e a altura o estipe (AE), com 25,91 e $28,56 \%$, respectivamente, sendo possivelmente as mais influenciadas pelo ambiente. Por outro lado, o comprimento da ráquis foliar (CRF) e o número de pares de folíolos (NPFO) foram as características morfológicas menos influenciadas pelas condições ambientais.

Todos os caracteres produtivos também mostraram valores de $\mathrm{F}$ significativos, diferindo apenas quanto ao nível de probabilidade (Quadro 2). Neste caso, apenas o peso da cabeça do palmito (PCP) e o diâmetro do meio do palmito (DMP) diferiram significativamente a $1 \%$ de probabilidade. Tais resultados evidenciam a ocorrência de variação entre plantas para todos os componentes de produção de palmito.

Entre os caracteres produtivos pode-se destacar o peso da cabeça do palmito, que exibiu média de $1.511,43 \mathrm{~g}$, o qual, após o processamento, correspondeu a apenas $16,47 \%$ do peso do palmito, alcançando 248,89 g de palmito total e 187,88 g de palmito creme.

Com exceção do número de toletes, todos os caracteres produtivos apresentaram coeficientes de variação elevados, demonstrando forte influência ambiental.

Quanto às estimativas de variâncias para os caracteres morfológicos, foi constatado que comprimento dos entrenós (CEN), comprimento da ráquis foliar (CRF), comprimento do folíolo central (CFC) e números de pares de folíolos (NPFO) apresentaram variâncias genéticas entre plantas maiores que as do erro, enquanto os demais exibiram variâncias genéticas entre plantas menores (Quadro 3). Há indícios de que as variações existentes entre as plantas para essas quatro características sejam decorrentes de expressão genética. Assim, se tais caracteres estiverem associados aos produtivos, podem tornarse úteis na seleção indireta de indivíduos promissores para palmito.

É importante ressaltar que, segundo Vencovsky (1977) e Falconer (1987), a variância devido às diferenças genéticas entre plantas não é só de natureza genética, pois além dela esta variação possui uma parte ambiental decorrente de diferenças verificadas entre plantas. Todavia, em estudos de correlações já realizados entre caracteres morfológicos e de produção de palmito em

R. Árvore, Viçosa-MG, v.27, n.4, p.435-442, 2003 pupunheira, a circunferência do estipe e o número de folhas têm sido mencionados, bem como as características morfológicas, de interesse ao melhoramento, por mostrarem associações positivas com os caracteres produtivos, por serem de fácil obtenção e, principalmente, por serem não-destrutíveis (Bovi et al., 1992; Bovi et al., 1993; Yuyama \& Costa, 1994). Entretanto, neste trabalho tais caracteres mostraram grande influência ambiental, levantando dúvidas sobre suas consistências na seleção de indivíduos desejáveis através de caracteres fenotípicos.

No que diz respeito aos coeficientes de repetibilidade, os caracteres morfológicos mostraram índices que variaram de $r=0,3044$ a $r=0,6716$ (Quadro 3). Os maiores valores foram registrados para o comprimento da ráquis foliar $(r=0,6716)$, comprimento dos entrenós $(\mathrm{r}=0,5488)$, número de pares de folíolos $(\mathrm{r}=0,5395)$ e comprimento do folíolo central $(\mathrm{r}=0,5317)$. Para Mansour et al. (1981), um caráter de considerável repetibilidade possui melhor desempenho que os demais, principalmente se apresentar alta associação com a variável desejável. Desta forma, pode-se supor que tais caracteres devam ter um bom controle genético, havendo possibilidades de proceder à seleção fenotípica simples indireta para produção de palmito.

Por outro lado, os caracteres número de folhas e circunferência do estipe foram as variáveis morfológicas que tiveram os menores coeficientes de repetibilidade, com $r=0,3044$ e $r=0,3412$, respectivamente, com praticamente as mesmas magnitudes. Esses baixos índices de repetibilidade podem ter ocorrido em função de os estipes cortados na mesma planta apresentarem desenvolvimentos distintos, em função de a emissão dos perfilhos ocorrer gradativamente, uma vez que os estipes da mesma planta são geneticamente iguais, por surgirem da mesma semente, funcionando como clones. Esses caracteres vêm auxiliando melhoristas na seleção de indivíduos desejáveis para palmito nesta palmeira, na seleção massal (Bovi et al., 1992, 1993). Com base nesses resultados, pode-se sugerir que esses caracteres sejam controlados por diferentes conjuntos gênicos e devam ser aplicados em métodos de melhoramento que apresentem controle parental, minimizando-se assim as influências ambientais.

É importante ressaltar que, com exceção do número de folhas e do comprimento da bainha foliar, as demais variáveis avaliadas nas folhas mostraram coeficiente de repetibilidade de médias magnitudes e que, embora sejam de difícil obtenção, são variáveis não-destrutivas que 
Quadro 2 - Análise da variância para oito caracteres de produção de palmito de 65 plantas de pupunheira da coleção de germoplasma da Embrapa Amazônia Oriental, Belém-PA, 2001

Table 2 - Variance analysis for eight heart palm production characters of 65 peach palm plants of Embrapa Eastern Amazon germ plasm collection, Belém, PA, 2001

\begin{tabular}{|c|c|c|c|c|}
\hline \multirow{2}{*}{ Caráter } & \multicolumn{2}{|c|}{ Quadrados Médios } & \multirow{2}{*}{ Média } & \multirow{2}{*}{$\begin{array}{l}\mathrm{CV} \\
(\%)\end{array}$} \\
\hline & Planta & Erro & & \\
\hline Peso da cabeça do palmito (g) & $695.416,4892 * *$ & $487.694,0804$ & $1.511,43$ & 46,20 \\
\hline Diâmetro do ápice do palmito ( $\mathrm{mm})$ & $58,6579 *$ & 37,9054 & 33,82 & 18,21 \\
\hline Diâmetro do meio do palmito (mm) & $69,5829 * *$ & 40,1230 & 28,15 & 22,50 \\
\hline Diâmetro da base do palmito $(\mathrm{mm})$ & $69,0298 *$ & 43,3648 & 29,79 & 22,10 \\
\hline Peso do palmito total $(\mathrm{g})$ & $2.3830,9008 *$ & $15.026,0846$ & 248,89 & 49,25 \\
\hline Peso do palmito creme $(\mathrm{g})$ & $14.608,1983^{*}$ & $11.249,8656$ & 187,88 & 56,45 \\
\hline Comprimento do palmito bruto $(\mathrm{cm})$ & $57,4131 *$ & 42,7153 & 36,03 & 18,14 \\
\hline Toletes de palmito (unid.) & $0,0410 *$ & 0,0297 & 2,11 & 8,15 \\
\hline
\end{tabular}

** significativo a $1 \%$ de probabilidade $\mathrm{e}^{*}$ significativo a $5 \%$ de probabilidade pelo teste $\mathrm{F}$.

Quadro 3 - Estimativas da variância genética entre plantas $\left(\sigma^{2}\right)$, variância do erro $\left(\sigma^{2}\right)$, coeficiente de repetibilidade $(r)$, coeficiente de determinação $\left(\mathrm{R}^{2}\right)$ para $\mathrm{k}$ repetições e número de estipes a serem cortados em uma seleção efetiva a $95 \%$ de certeza $(\eta)$, para oito caracteres morfológicos em pupunheiras, Belém-PA, 2001

Table 3 - Estimates of genetic variance among plants $\left(\sigma_{p}^{2}\right)$, environmental variance $\left(\sigma^{2}\right)$, repeatability coefficients $(r)$, determination coefficients $\left(R^{2}\right)$ for $k$ repetitions and stem number to be evaluated at an effective selection at $95 \%$ level ( $\eta$ ), for eight peach palm morphological characters, Belém, PA, 2001

\begin{tabular}{|c|c|c|c|c|c|}
\hline Caráter & $\sigma_{p}^{2}$ & $\overline{\sigma_{e}^{2}}$ & $\bar{r}$ & $\mathrm{R}^{2}$ & $\eta$ \\
\hline CAP $(\mathrm{cm})$ & 11,0348 & 21,3077 & 0,3412 & 41,34 & 37 \\
\hline $\mathrm{CEN}(\mathrm{cm})$ & 351,3773 & 288,8469 & 0,5488 & 57,58 & 19 \\
\hline $\mathrm{AE}(\mathrm{m})$ & 1,9968 & 2,4725 & 0,4468 & 50,18 & 23 \\
\hline NF (unid.) & 0,0744 & 0,1701 & 0,3044 & 37,92 & 43 \\
\hline $\mathrm{CBF}(\mathrm{cm})$ & 140,7836 & 201,5187 & 0,4113 & 47,36 & 27 \\
\hline $\mathrm{CRF}(\mathrm{m})$ & 0,0953 & 0,0466 & 0,6716 & 65,29 & 9 \\
\hline $\mathrm{CFC}(\mathrm{cm})$ & 45,2314 & 39,8441 & 0,5317 & 56,41 & 17 \\
\hline NPFO (unid.) & 0,3106 & 0,2651 & 0,5395 & 56,94 & 16 \\
\hline
\end{tabular}

CAP: circunferência à altura do peito; CEN: comprimento dos entrenós; AE: altura do estipe; NF: número de folhas; CBF: comprimento da bainha foliar; CRF: comprimento da ráquis foliar; CFC: comprimento do folíolo central; NPFO: número de pares de folíolo; e K: fator para diferentes números de medições $=2,6$.

podem estimar a produção de palmito. Em vista do exposto, acredita-se que possam ser úteis na seleção de indivíduos promissores para esta finalidade, com chances razoáveis de ganho genético.

Para Falconer (1987), uma das vantagens do coeficiente de repetibilidade é oferecer a possibilidade de conhecer a magnitude do coeficiente de herdabilidade $\left(h^{2}\right)$, por expressar o seu limite superior. Então, com base nos resultados aqui obtidos, pode-se esperar que dos caracteres morfológicos apenas o comprimento da ráquis foliar expresse alta herdabilidade e que todos os produtivos possuam baixas herdabilidades.
Os caracteres morfológicos variaram de 37,92 a $65,29 \%$ de certeza para expressar o real valor das plantas para o número de repetições efetuadas, estando mais da metade delas acima de 50\%. Dos caracteres avaliados, apenas o número de folhas, a circunferência do estipe e o comprimento da bainha foliar apresentaram baixas porcentagens, 37,92, 41,34 e 47,36\%, respectivamente. Tais resultados demonstram que o corte de três estipes por planta pode ser considerado de pouca confiabilidade.

No que diz respeito ao número de estipes a serem cortados por planta para efetuar uma seleção efetiva a $95 \%$ de certeza $(\eta)$, foi verificado que apenas o comprimento

R. Árvore, Viçosa-MG, v.27, n.4, p.435-442, 2003 
da ráquis foliar apresentou um número de medição possível de ser realizado nas pupunheiras avaliadas, ou seja, nove estipes/planta (Quadro 3). Os demais caracteres morfológicos exibiram números de medições elevados, necessitando de 16 a 43 estipes por planta para que os resultados tivessem consistência. Essas quantidades de medições tornam-se extremamente trabalhosas e até mesmo inviáveis de serem realizadas em pupunheiras com pouco perfilhamento, para produção de palmito, pois há a necessidade de deixar estipes na planta selecionada para dar continuidade às novas etapas do melhoramento.

Vale ressaltar que dos caracteres morfológicos o comprimento da ráquis foliar também apresentou as melhores estimativas para a variação genética entre plantas e para os coeficientes de repetibilidade e de determinação. Outro caráter que apresentou índice de repetibilidade de média magnitude, sendo também não-destrutivo e de fácil obtenção, foi o comprimento dos entrenós. Por outro lado, o número de folhas e a circunferência do estipe atingiram valores baixos, indicando ser necessário um grande número de medições para obter valores reais com $95 \%$ de certeza, apresentando, assim, irregularidades na repetição desses caracteres entre uma medição e outra na mesma planta.

O comprimento da ráquis foliar, o número de pares de folíolos e o comprimento do folíolo central, que mostraram ser medidas de difícil obtenção, fornecem indícios de exercerem influências indiretas na produção de palmito, pois este é formado exclusivamente por componentes foliares em estado de desenvolvimento. Para tais caracteres as repetibilidades alcançaram médias magnitudes, mostrando certa influência ambiental em suas expressões. Neste caso, em uma seleção fenotípica praticada para eles poderá ocorrer razoável eficiência, entretanto deve-se preferir o uso de métodos de melhoramento mais sofisticados, com um maior controle do efeito ambiental.

Todos os caracteres produtivos exibiram variâncias genéticas entre plantas inferiores às do erro (Quadro 4), evidenciando forte influência ambiental. Esses resultados estão condizentes com os obtidos por Gonçalves et al. (1982), Siqueira (1982), Costa et al. (1997), Ferreira et al. (1999) e Oliveira \& Fernandes (2000), que também constataram, em outros caracteres de produção, uma forte influência ambiental.

No caso dos coeficientes de repetibilidade, todos os caracteres produtivos exibiram baixas magnitudes, com valores inferiores a $\mathrm{r}=0,2202$ (Quadro 4). A variável peso do palmito creme (PPC) teve o menor índice de repetibilidade $(r=0,1030)$, seguida pelo comprimento do palmito bruto $(\mathrm{r}=0,1169)$ e pelo número de toletes $(\mathrm{r}=0,1192)$. Como todos os caracteres produtivos analisados estão diretamente relacionados à produção de palmito, pode-se sugerir que vários pares de genes devem influenciar suas expressões e, portanto, devem ter forte influência ambiental. Outros autores também verificaram

Quadro 4 - Estimativas da variância genética entre plantas $\left(\sigma^{2}\right)$, variância do erro $\left(\sigma^{2}\right)$, coeficiente de repetibilidade $(r)$, coeficiente de determinação $\left(R^{2}\right)$ para $\mathrm{k}$ repetições e número de estipes a serem cortados em uma seleção efetiva a $95 \%$ de certeza $(\eta)$, para oito caracteres produtivos em pupunheiras, Belém-PA, 2001

Table 4 - Estimates of genetic variance among plants $\left(\sigma^{2}\right)$, environmental variance $\left(\sigma^{2}\right)$, repeatability coefficients $(r)$, determination coefficients $\left(R^{2}\right)$ for $k$ repetition and stem number to be evaluated at an effective selection at $95 \%$ certainty level ( $\eta$ ) for eight peach palm productive characters Belém, PA, 2001

\begin{tabular}{|c|c|c|c|c|c|}
\hline Caráter & $\sigma_{\mathrm{p}}^{2}$ & $\sigma_{\mathrm{e}}^{2}$ & $\mathrm{r}$ & $\mathrm{R}^{2}$ & $\eta$ \\
\hline PCP $(\mathrm{g})$ & $79.893,2310$ & $487.694,0804$ & 0,1407 & 20,05 & 116 \\
\hline DAP $(\mathrm{mm})$ & 7,9817 & 37,9054 & 0,1739 & 24,08 & 90 \\
\hline DMP $(\mathrm{mm})$ & 11,3307 & 40,1230 & 0,2202 & 29,33 & 67 \\
\hline DBP $(\mathrm{mm})$ & 9,8711 & 43,3648 & 0,1854 & 25,42 & 83 \\
\hline PPT $(\mathrm{g})$ & $3.386,4677$ & $15.026,0846$ & 0,1839 & 25,24 & 165 \\
\hline PPC (g) & $1.291,6665$ & $11.249,8656$ & 0,1030 & 15,17 & 17 \\
\hline CPB (cm) & 15,6530 & 42,7153 & 0,1169 & 17,00 & 143 \\
\hline NTP (unid.) & 0,0040 & 0,0297 & 0,1192 & 17,31 & 140 \\
\hline
\end{tabular}

PCP: peso da cabeça do palmito; DAP: diâmetro do ápice do palmito; DMP: diâmetro do meio do palmito; DBP: diâmetro da base do palmito; PPT: peso do palmito total; PPC: peso do palmito creme; CPB: comprimento do palmito bruto; NTP: número de toletes de palmito; e K: fator para diferentes números de medições = 2,6. 
baixos coeficientes de repetibilidades em caracteres importantes na produção de plantas perenes, como a seringueira (Gonçalves et al., 1982; Vasconcelos et al., 1985; Gonçalves et al., 1990), o coqueiro (Siqueira, 1982), o cupuaçuzeiro (Fonseca et al., 1990; Costa et al., 1997), a alfafa (Fonseca et al., 1999) e o açaizeiro (Oliveira \& Fernandes, 2000).

Baixos coeficientes de determinação foram encontrados para todos os caracteres produtivos, sendo inferiores a 29,33\% (Quadro 4), e, desta forma, não oferecem credibilidade no corte de três estipes por planta. Portanto, há a necessidade de avaliações com um número bem maior de cortes do que os aqui praticados.

Em relação às variáveis produtivas, o número de medições necessárias para se ter $95 \%$ de predição do valor real nas plantas estudadas foi superior a 67 estipes, demonstrando ser impraticável o uso desses caracteres na seleção de indivíduos superiores para produção de palmito.

De modo geral, as baixas estimativas da variância genética entre plantas, do coeficiente de repetibilidade e do coeficiente de determinação obtida para os caracteres produtivos, ressaltam as dificuldades encontradas pelos melhoristas em identificar os melhores valores genotípicos a partir das análises fenotípicas obtidas, considerando apenas os estipes cortados $(\mathrm{k}=2,6)$ por planta. Como as predições dos valores reais foram baixas para esses caracteres, a superioridade ou inferioridade no comportamento das pupunheiras estudadas pode não ser mantida. Desta forma, há a necessidade de realizar medições adicionais para garantir maior confiabilidade ao processo de seleção. Outra alternativa seria melhorar as condições experimentais do local do estudo para detectar diferenças entre plantas com um menor número de medições.

\section{CONCLUSÕES}

1- Dos caracteres morfológicos, o comprimento da ráquis foliar possui maior repetibilidade e, conseqüentemente, valores elevados de herdabilidade poderão ser obtidos na avaliação dessa característica. Mas o comprimento dos entrenós de repetibilidade média, característica não-destrutiva e de fácil obtenção, pode ser importante na seleção de pupunheiras desejáveis para produção de palmito.

2- A circunferência do estipe e o número de folhas apresentam baixos índices de repetibilidades e não devem possuir regularidade na expressão para uso em seleção fenotípica de indivíduos superiores para palmito.

3- Todos os caracteres de produção de palmito apresentam coeficientes de repetibilidade negligíveis e necessitam de um grande número de medições para expressar o real valor das plantas a $95 \%$ de certeza.

4- A seleção com base em dados fenotípicos do comprimento da ráquis foliar deve ser eficaz na seleção de pupunheiras promissoras, se forem realizadas nove medições na mesma planta.

\section{REFERÊNCIAS BIBLIOGRÁFICAS}

BOVI, M. L. A.; SAES, L. A; GODOY JÚNIOR, G. Correlações fenotípicas entre caracteres não destrutíveis e palmito em pupunheiras. Turrialba, v. 42, n. 3, p. 382390, 1992.

BOVI, M. L. A. et al. Caracteres indiretos na seleção de pupunheiras inermes (Bactris gasipaes H.B.K.) para palmito. In: CONGRESSO INTERNACIONAL SOBRE BIOLOGIA, AGRONOMIA E INDUSTRIALIZACION DEL PIJUAYO, 4., 1993, Iquitos, Peru. Anais... Iquitos: [s.n.], p. 163-176, 1993.

CAMACHO, E. EL Pejibaye (Guilielma gasipaes H.B. K). In: Simpósio Internacional sobre plantas de interesse econômico de la flora Amazônica. Belém: ITCA, 1972. p. 101-106.

CLEMENT, C. R. Descritores mínimos para el pejybaye (Bactris gasipaes H.B.K.) y sus implicaciones

filogenéticas. 1986. $128 \mathrm{f}$. Tesis (Maestrea)-Universidad Costa Rica, Turrialba, 1986.

CLEMENT, C. R. A pupunha uma árvore domesticada. Ciência Hoje. v. especial. Amazônia, p. 66-73, 1991.

COSTA, J. G.; LEDA, A. S.; OLIVEIRA, M. N. Estimativa da repetibilidade de características de frutos de cupuaçuzeiro no Estado do Acre. Revista Brasileira de Fruticultura, v. 19, n. 3, p. 313-318, 1997.

CORNACCHIA, G.; CRUZ, C. D.; PIRES, I. E. Estimativa do coeficiente de repetibilidade para características fenotípicas de procedência de Pinus tecunumanii (Schw.) Eguiluz \& Perry e Pinus caribaea var. hondurensis Barret \& Golfari. Revista Árvore, v. 19, n. 3, p. 333-345, 1995.

CRUZ, C. D.; REGAZZI, A. J. Modelos biométricos aplicados ao melhoramento genético. Viçosa, MG: Universidade Federal de Viçosa, 1997. 390 p.

R. Árvore, Viçosa-MG, v.27, n.4, p.435-442, 2003 
FALCONER, D. S. Introdução à genética quantitativa. Viçosa, MG: Universidade Federal de Viçosa, 1987. 279 p.

FERREIRA, R. P. et al. Avaliação de cultivares de alfafa e estimativa de repetibilidade de caracteres forrageiros.

Pesquisa Agropecuária Brasileira, v. 34, n. 6, p. 995-1002, 1999.

FONSECA, C. E. L.; ESCOBAR, J. R.; BUENO, D. M. Variabilidade de alguns caracteres físicos e químicos do fruto do cupuaçuzeiro. Pesquisa Agropecuária Brasileira, v. 25 , n. 7, p. 1079-1084, 1990.

GONÇALVES, P. S.; ROSSETTI, A. G; PAIVA, J. R. Coeficiente de repetibilidade e eficiência do miniteste de produção na seleção de seringueira. Pesquisa

Agropecuária Brasileira, v. 17, n. 2, p. 233-237, 1982.

GONÇALVES, P. S.; CARDOSO, M.; SÁES, L. A. Estimativas de repetibilidade na seleção de árvores adultas de seringueira. Pesquisa Agropecuária Brasileira, v. 25, n. 7, p. 1031-1038, 1990.

MANSOUR, H.; NORDHEIM, E. V.; RUTLEDGE, J. J. Estimators of repetability. Theorical Applied Genetics, n. 60 , p. 151-156, 1981.
OLIVEIRA, M. S. P.; FERNANDES, G. L. C. Coeficiente de repetibilidade em característica de cacho do açaizeiro nas condições de Belém-PA. In: CONGRESSO BRASILEIRO DE FRUTICULTURA, 15, 2000, Fortaleza. Anais... Fortaleza: 2000.

SIQUEIRA, E. R. Coeficiente da repetibilidade da produção de frutos de coqueiro comum. Pesquisa Agropecuária Brasileira, v. 17, n. 3, p. 573-574, 1982.

SHRIKHANDE, V. J. Some consideration in designing experiments on coconut trees. Journal of Indian of Societes of Agricultural Statation , n. 9, p. 82-89, 1957.

YUYAMA, K.; COSTA, S. S. Estudo da altura do corte da pupunheira para extração de palmito. Revista Brasileira de Fruticultura, v. 16, n. 2, p. 77-82, 1994.

VASCONCELLOS, M. E. C. et al. Métodos de estimação do coeficiente de repetibilidade no melhoramento da seringueira. Pesquisa Agropecuária Brasileira, v. 20, n. 4, p. 433-437, 1985.

VENCOVSKY, R. Princípios de genética quantitativa. Piracicaba: Escola Superior de Agricultura "Luis de Queiroz”. 1977. 97 p. 